- Terahertz pulsed imaging was used to investigate the liquid transport kinetics of $\alpha$ alumina powder compacts.

- A range of compaction forces and sintering conditions were used for the terahertz measurements.

- The microstructure characteristic as well as the surface properties of the alumina compact have an impact on the water ingress rate.

- The mass transport mechanism of the water ingress into the alumina compacts is consistent with Darcy flow.

- The hydraulic radius of the alumina compacts was determined. 


\title{
Terahertz Pulsed Imaging as a New Method for Investigating the Liquid Transport Kinetics of $\alpha$-Alumina Powder Compacts
}

\author{
Mohammed Al-Sharabi ${ }^{\mathrm{a}}$, Daniel Markl ${ }^{\mathrm{b}, \mathrm{c}}$, Vincenzino Vivacqua ${ }^{\mathrm{d}}$, Prince \\ Bawuah $^{\mathrm{a}}$, Natalie MacLean ${ }^{\mathrm{b}}$, Marian Bentley ${ }^{\mathrm{a}}$, Andrew P.E. York ${ }^{\mathrm{e}}$, Michele \\ Marigo $^{d}$, Karen Huang ${ }^{\mathrm{d}}$, J. Axel Zeitler ${ }^{\mathrm{a}, *}$ \\ ${ }^{a}$ Department of Chemical Engineering and Biotechnology, University of Cambridge, \\ Cambridge, CB3 OAS, UK \\ ${ }^{b}$ Strathclyde Institute of Pharmacy and Biomedical Sciences, University of Strathclyde, 161 \\ Cathedral Street, G4 ORE Glasgow, UK \\ ${ }^{c}$ EPSRC Centre for Innovative Manufacturing in \\ Continuous Manufacturing and Crystallisation, University of Strathclyde, 99 George Street, \\ G1 1RD Glasgow, UK \\ ${ }^{d}$ Johnson Matthey Technology Centre, P.O. Box 1, Belasis Avenue, Billingham, Cleveland \\ TS23 $1 L B, U K$ \\ e Johnson Matthey Technology Centre, Blounts Court, Sonning Common, Reading, RG4 \\ $9 \mathrm{NH}, \mathrm{UK}$
}

\begin{abstract}
Investigating the liquid transport kinetics of solid catalysts is of great importance for gaining a better understanding of the manufacturing and performance of such catalysts during reaction upon contact with the liquid. Terahertz pulsed imaging (TPI) coupled with a newly designed flow cell was used to quantify the rate of water ingress into $\alpha$-alumina pellets with a range of different porosities. A wide range of compaction forces (cold compaction, $7 \mathrm{kN}$ to $58 \mathrm{kN}$ ) and sintering conditions (no firing and sintering at $1200^{\circ} \mathrm{C}$ and $1300^{\circ} \mathrm{C}$ ) was investigated to explore the optimal pellet microstructure, i.e. mechanically strong but sufficiently porous for fast liquid transport kinetics. The results confirm that both the microstructure characteristics, particularly porosity, as well as the surface properties, i.e. wettability, influence the liquid transport kinetics. Fitting the TPI penetration rates with a power law shows that the type of observed mass transport characteristics is consistent with Darcy flow. The Lucas-Washburn
\end{abstract}

\footnotetext{
${ }^{*}$ Corresponding author
} 
equation was used to calculate the hydraulic radius based on the transport data. In summary, the results demonstrate that TPI has great potential to study the liquid transport kinetics of porous ceramic catalysts and catalyst supports and that can comfortably quantify transport processes at rates of $250 \mathrm{\mu m} \mathrm{s}^{-1}$ and beyond in such substrates for better quality control and optimised design and performance of such materials.

Keywords:

Porous media, $\alpha$-alumina, terahertz pulsed imaging, microstructure, liquid transport, modelling

\section{Introduction}

Solid catalysts are used in a wide range of applications, such as steam reforming (Nichele et al., 2012, Bej et al., 2014), vehicle emissions control (Chen et al., 2018), ammonia synthesis (Zhou et al., 2013), Fischer-Tropsch synthesis (Louyot et al., 2018, Xu et al., 2020) and hydrogenation processes (Jadhav et al. 2014, Segobia et al. 2019; Li et al. 2020) to name a few. Such catalysts usually have a porous structure to maximise their surface area per unit of reactor volume (Sosna et al. 2020). Given that solvent transport plays a crucial role in the manufacturing as well as for the performance of the catalyst during reaction, liquid transport characteristics are of key importance in catalysis: it is an essential mechanism in wash-coating and impregnation processes during the manufacturing of catalysts (Nijhuis et al., 2001, Bowker et al., 2007), and it is a critical parameter for heterogenous catalysis, where the reactants and the catalyst have different phases, i.e. solid for the catalyst and liquid for the reactants (Schlögl, 2015).

A number of techniques have been used to study the mass transport characteristics of porous heterogenous catalysts, such as magnetic resonance imaging (MRI) (Koptyug et al., 2001; Lysova et al., 2005, Anadon et al., 2005; Gladden et al. 2006, Sankey et al., 2009; Lysova et al., 2010), nuclear magnetic resonance (NMR) (Weber et al., 2009; Mantle et al., 2010, D'Agostino et al. 
2012, 2013, Leutzsch et al. 2019) and gas chromatography (Zhokh \& Strizhak 2017). Techniques, such as MRI, are capable of providing quantitative and three-dimensional (3D) specially resolved information of multiphase displacement processes in porous media at a high-temporal resolution (?). In addition to these techniques, it is very useful to explore other methods that can provide valuable information on the mass transport properties of porous catalytic materials.

Terahertz pulsed imaging (TPI) is an analytical technique that can be used to resolve the liquid transport kinetics in porous media (Markl \& Zeitler, 2017) in a non-invasive and non-destructive manner. The principle of the TPI measurement for liquid transport is based on the concept initially introduced by Obradovic et al. (2007) to investigate the solvent ingress and swelling kinetics in solid blocks of polymer. This kind of measurement is conducted in a reflection setting where a pulse of terahertz radiation is focused on the sample. This incident terahertz pulse can penetrate a wide range of polymer and ceramic materials and is then reflected at every interface between two media with different refractive indices (Obradovic et al. 2007). Such an interface can be observed between the dry and wetted material when liquid penetrates into a porous medium. This liquid front typically indicates a change in refractive index and hence results in a reflection of the terahertz pulse (Yassin et al. 2015a). The reflection peak of the liquid front can then be tracked to study the transport kinetics of the liquid into the porous sample to study the impact of pore structure properties as well as the type of constituent materials on the liquid transport and swelling kinetics. In recent years, the method has been established in the pharmaceutical sciences to characterise tablets (Yassin et al. 2015b a; Markl et al., 2017b, 2018, Al-Sharabi et al., 2020).

In this work, the TPI methodology was further developed and applied to resolve the solvent transport in $\alpha$-alumina catalytic materials. TPI is a useful technique for studying the liquid transport in such ceramic catalysts as most of the ceramic materials are transparent to terahertz radiation (Zeitler, 2016. Jonuscheit, 2018). Given the relatively long wavelength of terahertz radiation 
( $0.1 \mathrm{~mm}$ to $3 \mathrm{~mm})$ very little scattering is expected from typical porous media that are used for heterogenous catalysis (Markl et al. 2017a). In addition, TPI is sensitive to small changes in the refractive index within the porous medium and therefore it is possible to resolve the liquid penetration kinetics due to subtle differences in the refractive indices of the liquid and dry sample (Yassin et al. 2015b a; Zeitler \& Shen, 2012). The high-time resolution due to the fast acquisition rate shows the potential of using TPI for resolving the rapid liquid transport kinetics of porous ceramic materials (?Markl \& Zeitler, 2017; Markl et al. 2018). However, the TPI method is currently providing one-dimensional (1D) information and further work can be conducted to further develop the technique and overcome such limitation. In industry, $\alpha$-alumina is used as a catalyst support for various processes, such as steam reforming (Yeboah et al. 1995) and Fischer-Tropsch catalysis (Rytter et al. 2019). It is important to study the liquid transport kinetics of alumina pellets prepared over a range of different compaction and firing conditions to explore whether a combination of sintering temperature and compaction pressure can produce a strong but still sufficiently porous pellet. At a given pellet density, there might also be a desirable microstructure which can be revealed by liquid penetration experiments.

This study aims to characterise the impact of the microstructure and surface properties of the alumina powder compacts with varying compaction and heat treatment conditions on the liquid penetration kinetics.

\section{Materials and Methods}

\subsection{Materials and Sample Preparation}

Flat-faced, cylindrical pellets were made from a mixture of mainly $\alpha$-alumina powder with $5 \% \mathrm{~m} / \mathrm{m} \max$ of polymeric organic additives and $1 \% \mathrm{~m} / \mathrm{m}$ max of inorganic additives (Granalox ${ }^{\circledR}$ NM 99, Nabaltec AG, Schwandorf, Germany). These powder compacts were prepared via direct compression using a compaction simulator (Styl'One Evolution, Medelpharm, Beynost, France). The samples have a diameter and thickness of around $11.28 \mathrm{~mm}$ and $2 \mathrm{~mm}$, respec- 
Table 1: Nominal porosity, $f_{\text {nominal }}(\%)$, of the samples used for the TPI measurements and its standard deviation. The average $f_{\text {nominal }}$ was determined based on 10 samples per batch.

\begin{tabular}{lcccc}
\hline Compaction force $(\mathrm{kN})$ & 7 & 23 & 40 & 58 \\
\hline Unfired & $39.18 \pm 0.12$ & $36.09 \pm 0.17$ & $34.57 \pm 0.06$ & $33.46 \pm 0.10$ \\
Fired at $1200^{\circ} \mathrm{C}$ & $36.12 \pm 0.22$ & $33.00 \pm 0.18$ & $31.44 \pm 0.19$ & $30.29 \pm 0.13$ \\
Fired at $1300^{\circ} \mathrm{C}$ & $27.79 \pm 0.30$ & $24.61 \pm 0.13$ & $22.97 \pm 0.21$ & $21.93 \pm 0.21$ \\
\hline
\end{tabular}

tively. The diameter and thickness were kept constant while the filling weight of the powder material was adjusted to vary the pellet porosity. The pellets were compacted at compression forces of $7 \mathrm{kN}, 23 \mathrm{kN}, 40 \mathrm{kN}$ and $58 \mathrm{kN}$ yielding four classes of samples with different porosities. These samples will be referred to as unfired samples since no heat treatment was performed after the compaction process.

In addition to the set of unfired samples two further sets of pellets were prepared by identical compaction conditions but subsequently fired at temperatures of $1200{ }^{\circ} \mathrm{C}$ or $1300{ }^{\circ} \mathrm{C}$ with a ramp rate of $200{ }^{\circ} \mathrm{Ch}^{-1}$ and a dwell time of $6 \mathrm{~h}$. The samples were then cooled down to room temperature at a cooling rate of approximately $100{ }^{\circ} \mathrm{Ch}^{-1}$. The organic polymeric additive was completely pyrolised during the firing process and sintering occurred. As a result the samples were shrunk in thickness and diameter after firing, which in turn resulted in a reduction of the pellet porosity compared to the unfired samples.

For the 12 batches of varying porosities (Table 1), three pellets per batch were measured using TPI to obtain an average time-resolved liquid penetration profile of each sample condition.

\subsection{Calculation of Nominal Porosity}

The nominal porosity, $f_{\text {nominal }}$, was determined using the relative density, i.e. solid fraction, as described in Equation 1 (Stirnimann et al., 2013, Markl et al. 2017a). The relative density is the ratio of the apparent density to the true density, $\rho_{\text {true }}$, of the powder compact. The apparent density of the sample was calculated from the physical dimensions of the sample, i.e. the thickness, $H$, diam- 
eter, $D$, and weight, $W$. The true density of the alumina powder was measured using helium pycnometry (Micromeritics ACCUPYC 1340, Austin (TX), USA). These measurements were conducted after the compaction and firing of the pellets. The true densities were $(3.642 \pm 0.015) \mathrm{g} \mathrm{cm}^{-3},(3.822 \pm 0.006) \mathrm{g} \mathrm{cm}^{-3}$ and $(3.838 \pm 0.025) \mathrm{g} \mathrm{cm}^{-3}$ for the unfired, $1200{ }^{\circ} \mathrm{C}$ and $1300{ }^{\circ} \mathrm{C}$ batches, respectively. The true density of each batch is an average of the true densities of the 4 compression forces, i.e. $7 \mathrm{kN}, 23 \mathrm{kN}, 40 \mathrm{kN}$ and $58 \mathrm{kN}$.

$$
f_{\text {nominal }}(\%)=\left(1-\frac{4 W}{\pi D^{2} H \rho_{\text {true }}}\right) \times 100
$$

\subsection{In-Situ Monitoring of Liquid Transport}

\subsubsection{Experimental Setup}

TPI measurements were conducted to investigate the 1D transport kinetics of the alumina samples. The setup used for the in-situ TPI measurements was composed of a bespoke flow cell (Figure 1) and a commercial terahertz system (TeraPulse 4000, Teraview Ltd., Cambridge, UK) combined with a fiber-based reflection probe that had an $18 \mathrm{~mm}$ focal length silicon lens. The probe head was on a linear scale for ease of adjustment. The beam resulting of the $\mathrm{THz}$ optics had a beam waist of around $1 \mathrm{~mm}$ at the focus with an incident angle of $13^{\circ}$.

The flow cell (Figure 1) was specifically designed for conducting liquid transport measurements using TPI while considering easy handling of the sample, better sealing and enhanced data acquisition quality compared to the previous model (Yassin et al. 2015a; Markl et al., 2018). The body of the flow cell and the sample holder were made of 316 stainless steel to avoid corrosion upon contact with the liquid and hence increase the lifetime of the flow cell. The threaded window (Figure 1) is used to close the flow cell from the top. Since the terahertz radiation propagates through this window to reach the sample, the window was made of high-density polyethylene (HDPE) which is semi-transparent to terahertz radiation and thus causes minimum signal loss. The window has a channel 
c)

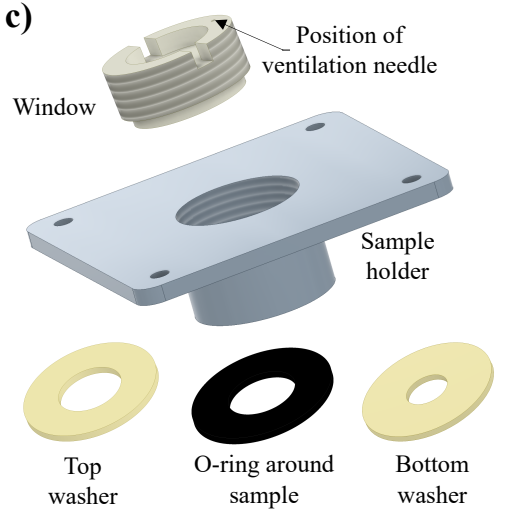

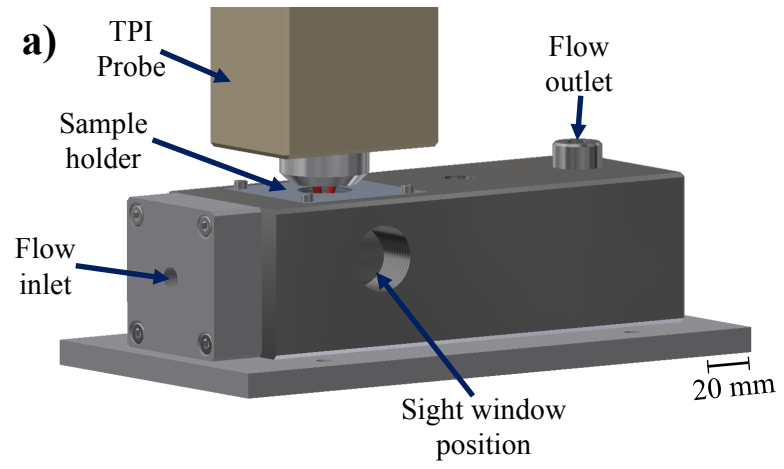

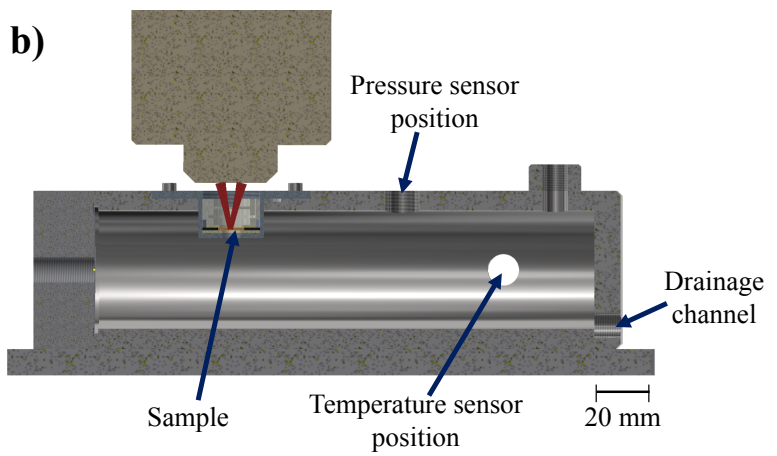

d)

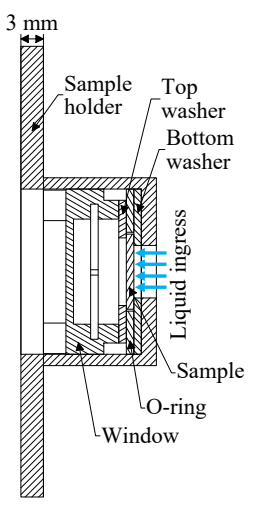

Figure 1: Flow cell: (a) and (b) The flow cell used for studying the liquid transport kinetics of the alumina compacts using TPI. Schematic of the sample holder: (c) 3D schematics of the window, sample holder and washers that are used for holding and sealing of the sample during the TPI measurement; (d) a sub-view of the cross-section of the setup. 
for a ventilation needle, as illustrated in Figure 1, to allow the removal of the air trapped between the tablet and the window after closing the flow cell. This ventilation is required to avoid a two-phase flow as air needs to escape the system upon the transport of the liquid into the pores of the sample. The flow cell was fitted with a sight window to monitor the liquid level during filling. In addition, the flow cell is equipped with sensors to measure the temperature and the pressure of the liquid filling the flow cell.

The sample was placed in the sample holder, which was fitted to the flow cell. $1 \mathrm{~mm}$ thick polytetrafluoroethylene (PTFE) washers were used beneath and on the top of the sample and a nitrile rubber O-ring (varying sizes based on the sample dimensions) was used around the sample to provide the required sealing and ensure the sample is stationary during the measurement as illustrated in Figure 1. The flow cell was closed by screwing a threaded window until it pushes on the top washer to provide the required sealing. The position of the TPI probe was adjusted in all directions to set the focus of the terahertz beam on the centre of the pellet and maximise the peak to peak intensity in the terahertz waveform.

Three containers were used for the inlet flow, outlet flow and drainage, respectively (Figure 2). It was ensured that the tubes were securely connected to the channels of the flow cell and were fixed to the containers avoiding the movement of the tubes off the containers. The container for the inlet flow was filled with water at a temperature of $20^{\circ} \mathrm{C}$. The outlet flow container was filled with a small amount of water to monitor the removal of the air bubbles from the flow cell.

\subsubsection{Experimental Procedure}

Prior to starting the experiment, a baseline, i.e. noise background when measured into air without reflection, and reference, i.e. reflection from a mirror, were acquired. 500 waveforms were co-averaged for both the baseline and the reference at an acquisition rate of $15 \mathrm{~Hz}$ with a total time-delay of $45 \mathrm{ps}$ for the $\mathrm{THz}$ waveform.

The inlet and outlet valves were opened while the drainage valve was closed 


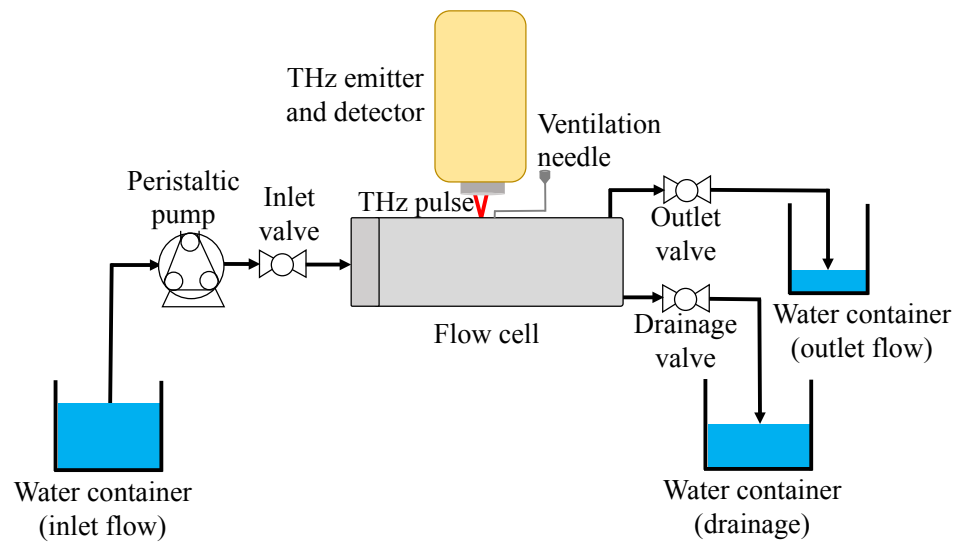

Figure 2: The experimental setup for the in-situ TPI measurements of the alumina pellets.

at the start of each measurement. The pump was turned on and water was pumped into the flow cell at a flow rate of $13 \mathrm{ml} \mathrm{min}^{-1}$ to displace the air in the flow cell with water. The data was acquired once the water level was about to reach the front face of the sample. The water level was monitored through the sight window. TPI data was acquired continuously to capture the dynamic process of water ingress into the sample at an acquisition rate of $15 \mathrm{~Hz}$ with a total time-delay of $45 \mathrm{ps}$ for the $\mathrm{THz}$ waveform. The water movement through the sample is observed in the $\mathrm{THz}$ waveform as illustrated in Figure 3 . The measurement was stopped and the pump was turned off once the sample was fully hydrated, i.e. water reached the back face of the sample and caused the magnitude of the amplitude of the back face refection peak to increase. The flow cell was drained by opening the drainage valve and was cleaned thoroughly prior conducting a new sample measurement.

\subsubsection{Processing of TPI Data}

The raw TPI data were first deconvolved by dividing the Fourier transformed raw signal of the sample, $\operatorname{FFT}[s(t)]$, by the Fourier transformed reference signal, $\operatorname{FFT}[r(t)]$. A double Gaussian band-pass filter, $\operatorname{FFT}\left[f_{\mathrm{DG}}(t)\right]$ was used in frequency-domain (FD) according to Equations 2 and 3 (Zeitler \& Shen, 2012 Markl et al. 2018). The pulse width was defined by the high frequency, HF, and 

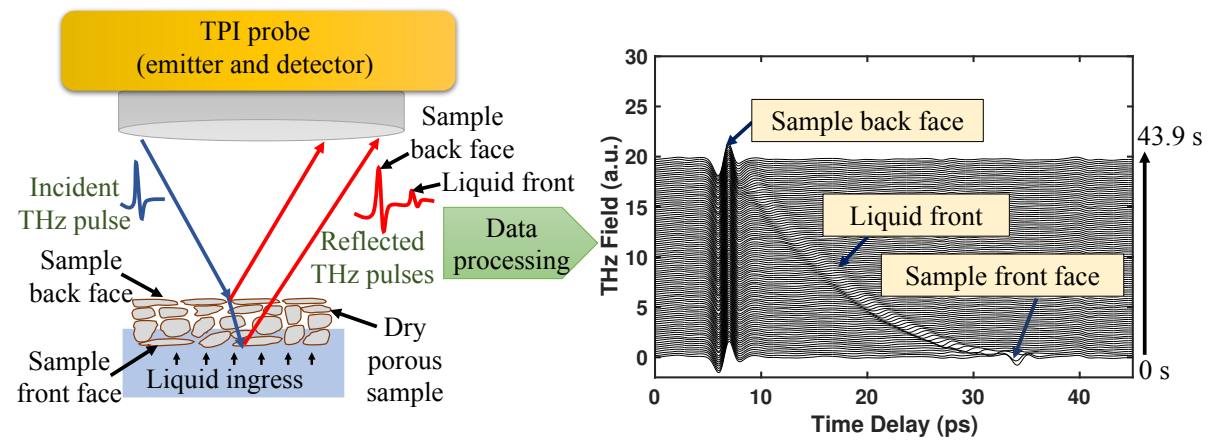

Figure 3: A schematic showing how the TPI method is used to investigate the liquid penetration kinetics of porous powder compacts. The waterfall plot shows the ingress of water into an unfired alumina pellet compacted at $7 \mathrm{kN}$. Each seventh deconvolved waveform was plotted with an offset of 0.03 a.u. between each deconvolved waveform, where the first measurement is represented by the first waveform at the bottom of the waterfall plot.

low frequency, LF, limits of the double Gaussian band-pass filter (Markl et al. 2018). An inverse Fourier transform was then used to calculate the deconvolved time-domain waveform, $h(t)$.

$$
\begin{aligned}
h(t) & =\mathrm{FFT}^{-1}\left[\frac{\mathrm{FFT}[s(t)]}{\operatorname{FFT}[r(t)]} \times \mathrm{FFT}\left[f_{\mathrm{DG}}(t)\right]\right] \\
f_{\mathrm{DG}}(t) & =\frac{1}{\mathrm{HF}} \exp \left(-\frac{t^{2}}{\mathrm{HF}^{2}}\right)-\frac{1}{\mathrm{LF}} \exp \left(-\frac{t^{2}}{\mathrm{LF}^{2}}\right)
\end{aligned}
$$

where $t$ is the optical time delay.

The reflection peak of the liquid front was then tracked in $h(t)$ for each sample to determine the time-resolved liquid penetration profiles of each batch. The data processing was implemented in MATLAB (MathWorks, Massachusetts, USA). The total number of pixels representing the thickness of the sample, $N_{\text {total }}$, was first extracted by subtracting the indices of the positive peak of the back face from the negative peak of the front face of the dry sample. The penetration depth of liquid, $y$, as a function of time, $t$, was then calculated using the following equation

$$
y(t)=N(t) \frac{H}{N_{\text {total }}}
$$


where $N(t)$ is the index of the water front peak relative to the front face of the sample in the $\mathrm{THz}$ waveform at a specific time. Given that the terahertz waveforms were acquired at an acquisition rate of $15 \mathrm{~Hz}$ without averaging the waveforms, the time step, $t_{\mathrm{s}}$, between each two $\mathrm{THz}$ waveforms is $\frac{1}{15} \mathrm{~s}$. $t$ at a given penetration depth was then calculated as

$$
t=W_{\mathrm{n}} t_{\mathrm{s}}
$$

where $W_{\mathrm{n}}$ is the waveform number at a specific penetration depth.

The liquid penetration profiles were further analysed using the Lucas-Washburn equation.

\subsubsection{Liquid Penetration Model}

The Lucas-Washburn equation describes an analytical model that expresses the position of the liquid penetration front in a porous medium as a function of time (Washburn, 1921). This model is a combination of the Young-Laplace and Hagen-Poiseuille equations, which can be used to describe Darcy flow; an important mass transport mechanism that is governed by a capillary pressure gradient throughout the porous medium (Markl \& Zeitler, 2017). In this formalism, the pore space is assumed to consist of cylindrical and uniform capillary tubes (Cummins et al. 2017) as illustrated in Figure 4. Hence, the penetration depth of a liquid, $y$, with a surface tension, $\gamma$, and a dynamic viscosity, $\eta$, through a porous system as a function of time, $t$, for a laminar flow can be described as follows (Markl et al., 2018)

$$
y(t)=\sqrt{\frac{R_{\mathrm{h}, \mathrm{eq}} \gamma \cos \theta t}{2 \eta}},
$$

where $\theta$ is the contact angle between the liquid and the solid material and $R_{\mathrm{h}, \text { eq }}$ is the hydraulic radius.

\subsection{Mercury Porosimetry}

Mercury intrusion/extrusion measurements were conducted using a Micromeritics AutoPore 9520 mercury porosimeter. The measurements were performed 

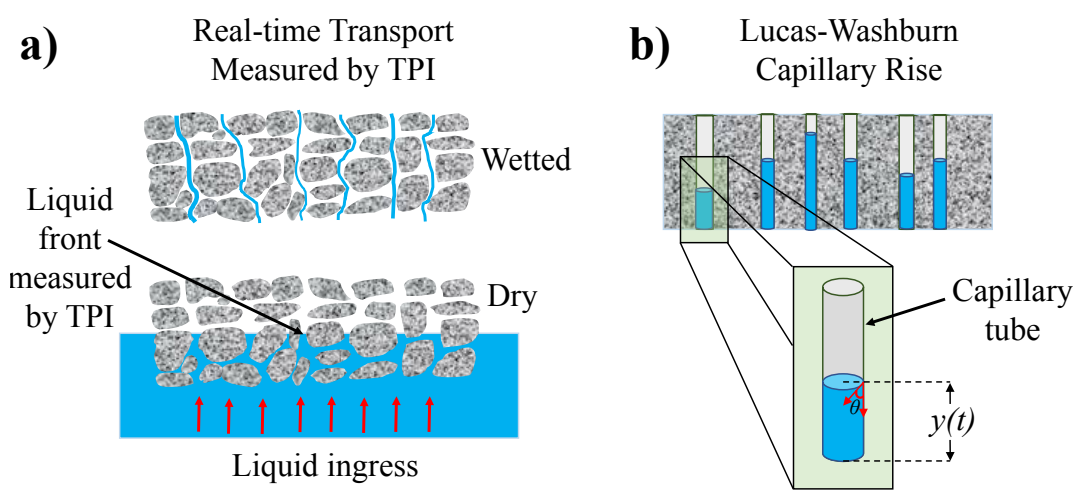

Figure 4: A schematic of the liquid transport into a porous medium a) measured by TPI and b) modelled using Lucas-Washburn Equation.

in accordance with ASTM D4284-03 (Standard Test Method for Determining Pore Volume Distribution of Catalysts by Mercury Intrusion Porosimetry). Intrusion measurements were conducted over the pressure range of $3.45 \times 10^{3} \mathrm{~Pa}$ to $413.69 \times 10^{6} \mathrm{~Pa}$ followed by extrusion down to atmospheric pressure. An equilibrium time of $15 \mathrm{~s}$ was set for each data point on both the intrusion and extrusion curves. The surface tension and the contact angle of mercury were selected as $485 \mathrm{mN} \mathrm{m}^{-1}$ and $140^{\circ}$, respectively. The samples were dried at $115^{\circ} \mathrm{C}$ overnight in an oven prior to analysis. The effects of temperature and pressure that manifested during the porosimetry run were accounted for by blank correction runs on empty penetrometer tubes which were subsequently subtracted from the experimental data.

\subsection{Contact Angle Measurements}

Measuring the contact angle, $\theta_{c}$, is a common method to quantify the wettabililty of a solid material (Jose \& Alagar, 2015, Dwivedi et al., 2017). The wettabiliy depends on the properties of both the liquid as well as the solid material (Kulkarni \& Shaw, 2016). Smaller values of the contact angle indicate greater wetting of the solid (Dwivedi et al. 2017). In the case of water, a contact angle with a value of $>90^{\circ}$ indicates poor wettability, whereas a contact angle with a value of $<90^{\circ}$ indicates high wettability (Hornyak \& Rao, 2016). 
The contact angle measurements of alumina samples were conducted with water using a drop shape analyser (Krüss DSA30, Krüss GmbH, Hamburg, Germany). Video recordings were taken at a rate of 30 frames per second as a droplet of purified water (Milli-Q IQ 7000, Merck Millipore, Watford, UK) was dispensed on to the surface of the tablet. MATLAB (MathWorks, Massachusetts, USA) was used to extrapolate contact angle-time profiles. The initial contact angle, $\theta_{c, 0}$, was calculated by fitting the contact angle profiles with a two-phase exponential decay model using GraphPad Prism 8 (GraphPad Software LLC, San Diego, USA).

$$
\begin{aligned}
\theta_{c}(t) & =\theta_{c, p}+s_{f} e^{-k_{f} \cdot t}+s_{s} e^{-k_{s} \cdot t} \\
s_{f} & =\theta_{c, 0} \cdot x_{f s} \\
s_{s} & =\theta_{c, 0}\left(1-x_{f s}\right)
\end{aligned}
$$

$\theta_{c, 0}$ and $\theta_{c, p}$ are the contact angles at initial and infinite time, respectively. $k_{f}$ and $k_{s}$ are the rate constants for the fast and slow phases, respectively. The fraction of time dominated by the fast phase of the reaction is described as $x_{f s}$.

The contact angle of three pellets per batch were measured.

\section{Results and Discussion}

\subsection{In-situ Monitoring of Liquid Transport}

Figure 5 shows the waterfall plots of the unfired alumina samples at the four different compaction forces of $7 \mathrm{kN}, 23 \mathrm{kN}, 40 \mathrm{kN}$ and $58 \mathrm{kN}$ to highlight the effect of the change in porosity due to the different compaction forces on the liquid transport kinetics. The TPI results show that the porosity clearly influences the rate at which water ingresses into the alumina compacts. The time to fully wet the sample, as indicated by the arrows on the right of the waterfall plots, shortens with decreasing compaction force and hence increasing the porosity. The same behaviour was also observed when comparing the dif- 
ferent compaction forces within each set of samples, at each firing temperature, i.e. $1200{ }^{\circ} \mathrm{C}$ and $1300{ }^{\circ} \mathrm{C}$ (Figures A.12 and A.13 in Appendix A).

The relative difference between the refractive indices of the two media through which the terahertz pulse propagates determines the amplitude of the reflection peaks observed in the terahertz waveform (Zeitler, 2016). The reflection peak amplitude is related to the reflection coefficient, $r_{12}$, which is described in Equation 8 based on Verdet convention, for a terahertz pulse travelling from a medium with a refractive index of $n_{1}$ to a medium with a refractive index of $n_{2}$ (Markl et al., 2018).

$$
r_{12}=\frac{n_{2}-n_{1}}{n_{1}+n_{2}}
$$

The sign of the pulse amplitude indicates whether the pulse travels into or out of a medium of higher or lower refractive index. Here, the terahertz pulse travels from air $\left(n_{\text {air }}=1\right)$ into the alumina pellet $\left(2<n_{\text {eff }}<3\right.$ at $\left.1 \mathrm{THz}\right)$ and back to air before the water has reached the sample. Therefore, a positive peak is observed for the back face of the pellet while a negative peak is observed for the front face of the pellet before wetting according to Equation 8 . The back face refers to the top part of the pellet while the front face refers to the bottom part of the pellet facing the water as illustrated in Figure 3. For a non-dissolving material, such as alumina, the magnitude of the amplitude of the water reflection peak is governed by the relative difference between the refractive indices of the wetted and the dry material. The wetted alumina has a higher refractive index compared to that of the dry alumina due to the replacement of air in the pores with water, which has a higher refractive index compared to that of air $\left(n_{\text {water }} \approx 2.1\right.$ at 1 $\mathrm{THz}$ (Pickwell \& Wallace, 2006)). Therefore, the reflection peak from the liquid front is positive in the waterfall plot.

The waterfall plots show that the alumina pellets do not disintegrate in water as the positive reflection peak from the back face of the sample remains after the full wetting of the sample. These plots also show that the alumina samples are non-swelling upon contact with water as the reflection peak from the sample 
back face remains in the same temporal position, which indicates that there is no change in the thickness of the sample as opposed to swelling systems (Obradovic et al., 2007, Yassin et al., 2015b a). However, the magnitude of this positive peak from the sample back face increases once the water has reached the back face. This is attributed to the fact that the displacement of air in the pores of the compact by water increases the effective refractive index of the sample and hence results in a larger relative difference between the air and the back face of the wetted sample. This in turn increases the amplitude of the reflection peak at the interface of the air/wetted alumina pellet.

The waterfall plots in Figure 6 highlight the impact of the change in porosity due to firing on the rate of water ingress into the alumina samples at the same compaction force of $7 \mathrm{kN}$. The porosity of the pellet decreases with an increase in the firing temperature due to an increase in shrinkage of the pore space, i.e. pore volume, upon subjecting the sample to a higher firing temperature. Therefore, the time to fully wet the sample, as indicated by the arrows on the right of each waterfall plots, extends with increasing firing temperature at the same compaction force.

However, although the unfired samples have the highest porosity compared to the fired samples at the same compaction force, the total duration of the full wetting of the unfired samples, as indicated by the arrows on the right of the waterfall plots, is larger. The same behaviour was also observed when comparing the duration of water ingress into the unfired and fired samples at the other compaction forces of $23 \mathrm{kN}, 40 \mathrm{kN}$ and $58 \mathrm{kN}$. The cause of the larger total duration of the full wetting of the unfired samples by water compared to the fired samples at the same compaction force is justified in the following sections.

\subsection{Quantitative Analysis of the Liquid Transport Process}

The averaged liquid penetration profiles, i.e. penetration depth as a function of time, of the alumina samples are presented in Figure 7 . The small standard deviation of the liquid penetration profiles of the alumina samples show the reli- 

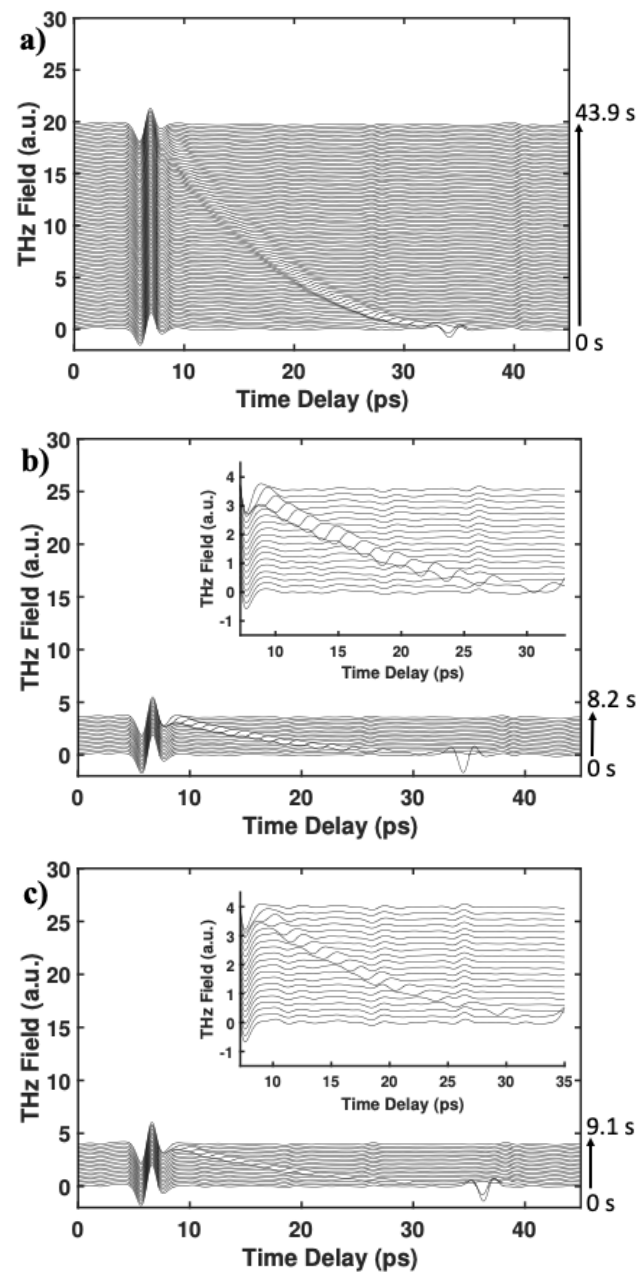

Figure 6: Waterfall plots visualising the water ingress into the alumina samples compacted at the same force of $7 \mathrm{kN}$ and with different firing temperatures: (a) Unfired (b) fired at $1200^{\circ} \mathrm{C}$ and (c) fired at $1300^{\circ} \mathrm{C}$. Each seventh deconvolved waveform was plotted with an offset of 0.03 a.u. between each deconvolved waveform. The arrow on the right of each waterfall plot indicates the duration of the full wetting of the sample by water in seconds. 
ability of using the TPI method to investigate the rate of the liquid penetration into such porous media. The liquid penetration profiles indicate that the rate of water transport increases with decreasing compaction force within each set of temperature condition (i.e. unfired or fired). This is clearly due to the fact that compacting the powder at a lower force results in a higher porosity of the sample. The rate of water transport decreases as the firing temperature increases for each compaction force due to a decrease in porosity upon increasing the firing temperature. Although the unfired samples have the highest porosity, such samples have the slowest rate of liquid transport in comparison with the fired samples at the same compaction force. This can be attributed to the different surface properties of the unfired and fired samples. The unfired samples contain polymeric additives, which are removed during the firing process of the alumina pellets. The presence of such additives in the unfired samples have an effect on the wettability of the pellet and hence its liquid transport rate. Therefore, contact angle measurements were conducted to investigate the wettability of the unfired and fired samples and confirm whether the slower transport rates of the unfired samples, observed in the TPI results, are due to differences in the surfaces properties of the unfired and fired pellets. Beside the porosity and wettability properties of the pellet, the change of other pore structure characteristics of the pellet, such as pore connectivity, during firing is speculated to also have an impact on the liquid penetration kinetics.

\subsection{Wettability Analysis}

Contact angle was used to quantify the wettability of the alumina samples by water. Figure 8 shows that the values of the initial contact angle, $\theta_{c, 0}$, for the unfired samples are larger than those for the fired samples. This indicates that the unfired samples have lower wettability, presumably due to the presence of the polymeric binder, compared to the fired samples at each compaction force. Therefore, the results of the contact angle measurements explain the slower transport kinetics of the unfired samples observed in the terahertz measurements. These findings show that, beside porosity, the difference in the surface 

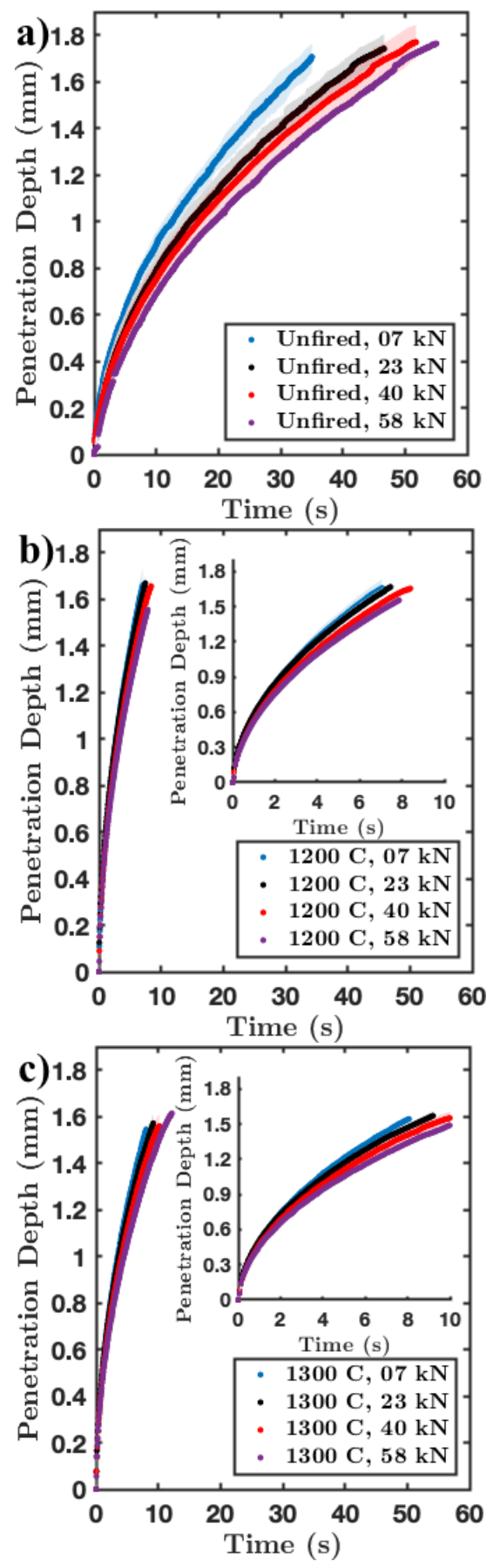

Figure 7: The average liquid penetration depth as a function of time for (a) the unfired alumina samples, and the alumina samples fired at (b) $1200{ }^{\circ} \mathrm{C}$ and (c) at $1300{ }^{\circ} \mathrm{C}$. The standard deviation for each profile is represented by the shaded area. Three samples per batch were used to determine the standard devlation. 


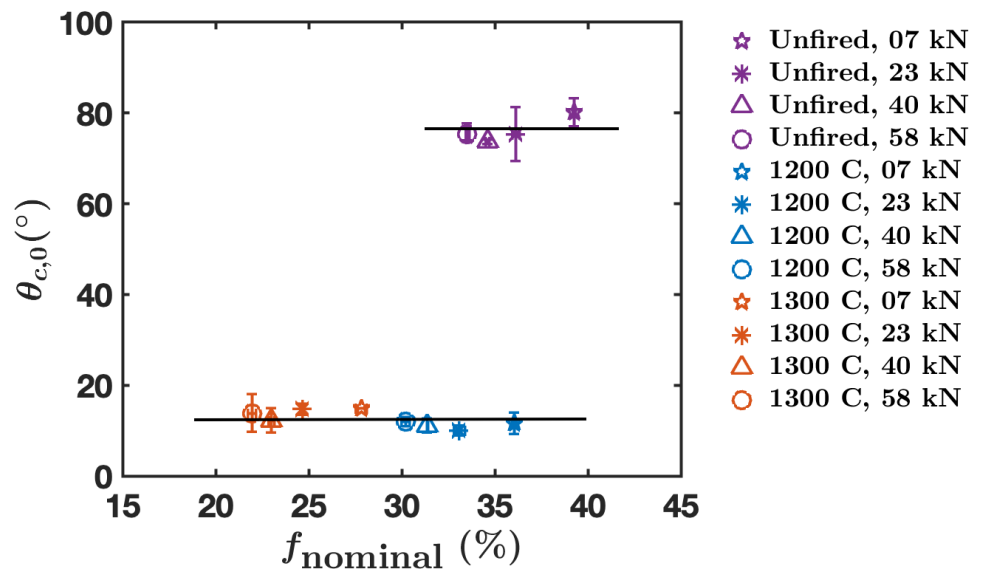

Figure 8: The initial contact angle, $\theta_{c, 0}$, of the alumina samples as a function of the nominal porosity, $f_{\text {nominal }}$. The black lines are added to guide the eye.

properties due to the presence of different ingredients in the porous system has an impact on the liquid transport kinetics.

\subsection{Analysis of Liquid Transport Kinetics}

A simple power law, described in Equation 9, can be used to model the liquid transport kinetics of the alumina samples, as previously used to analyse the liquid transport kinetics of pharmaceutical tablets (Yassin et al., 2015b. Markl et al., 2018).

$$
y(t)=k t^{m}
$$

where $k$ is a fitting parameter describing the rate of liquid transport and the exponent $m$ defines the type of the mass transport mechanism (Yassin et al. 2015a). The transport process can either be considered as Darcy flow (driven by a gradient in the capillary pressure) for an $m$ value of 0.5 or anomalous transport for $0.5<m<1$ (Yassin et al., 2015b; Markl \& Zeitler, 2017).

The power law was fitted to the experimental liquid penetration data of each pellet as illustrated in Figure A.15 (see Appendix A). The fitting parameters are summarised in Figure 9, which indicate that $k$ increases with an increase in 
the porosity of the sample within each temperature condition, i.e. unfired and fired. However, the values of the $k$ parameter for the unfired samples are lower than those of the fired samples at the same compaction force due to the presence of the polymeric additives which results in a reduction in the mass transport rate. The exponent $m$ for the different batches of the alumina pellets has a value around 0.5 as shown in Figure 9 and hence the mass transport mechanism can be considered to obey Darcy flow. Using the Lucas-Washburn equation the hydraulic radius of the alumina pellets can be calculated using this transport data.

Mercury porosimetry was used to better understand the pore size of the alumina samples and how the pore size and its distribution influence the transport kinetics through investigating the relationship between the $k$ parameter obtained from the terahertz liquid transport experiments and the pore size measured by the mercury porosimetry. Figure 10 shows that not only the pore size correlates with porosity but also with the $k$ parameter. It can be seen from the data plotted in Figure 10 that the median pore diameter, obtained from the mercury porosimetry measurements, decreases with an increase in the compaction force, i.e. a decrease in the porosity, within each set of temperature condition. The pores seem to get larger upon firing when comparing the unfired and fired samples at the same compaction force. It is speculated that such observation can be attributed to the removal of the polymeric additives as well as forging smaller pores into one larger pore during the sintering of the unfired samples. Further firing of the pellet, i.e. increasing the firing temperature, results in the shrinking of the pores which explains the decrease in the median pore diameter upon increasing the firing temperature when comparing the fired sets of samples at the same compaction force. The value of the $k$ parameter increases with an increase in the median pore diameter within each set of temperature condition. It is important to note that the pores measured by the mercury porosimetry are the open pores in the alumina powder compacts. However, it is possible for closed pores to be formed in such compacts upon firing. The median pore diameter $(906 \AA$ to $1128 \AA$ for the unfired alumina samples and $1029 \AA$ to $1291 \AA$ for 


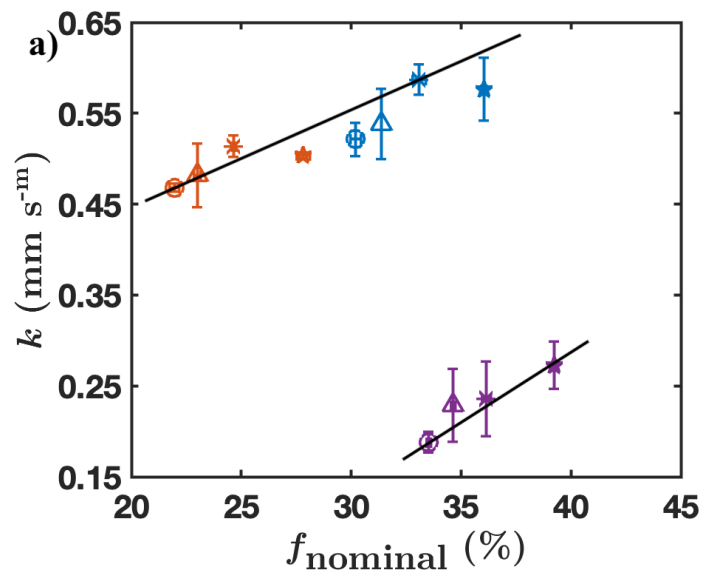

นnfired, $07 \mathrm{kN}$

* Unfired, $23 \mathrm{kN}$

$\triangle$ Unfired, $40 \mathrm{kN}$

○ Unfired, $58 \mathrm{kN}$

4 $1200 \mathrm{C}, 07 \mathrm{kN}$

* $1200 \mathrm{C}, 23 \mathrm{kN}$

$\triangle 1200 \mathrm{C}, 40 \mathrm{kN}$

○ $1200 \mathrm{C}, 58 \mathrm{kN}$

^ $1300 \mathrm{C}, 07 \mathrm{kN}$

* $1300 \mathrm{C}, 23 \mathrm{kN}$

$\triangle 1300 \mathrm{C}, 40 \mathrm{kN}$

○ $1300 \mathrm{C}, 58 \mathrm{kN}$

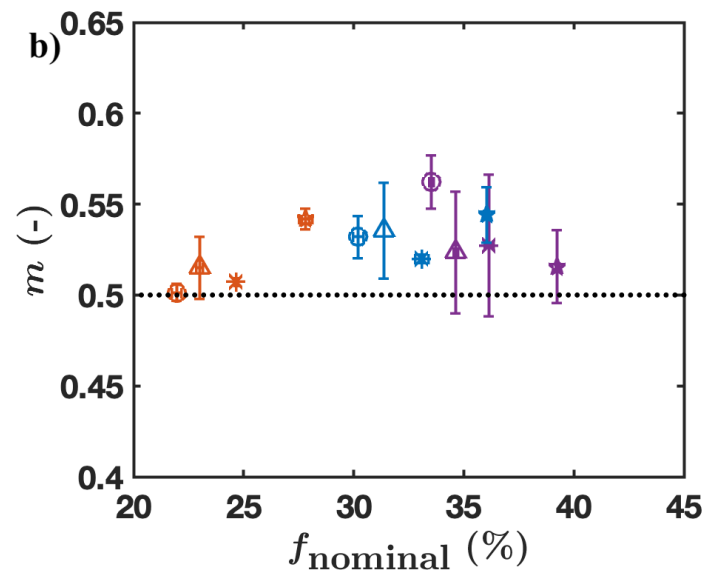

Figure 9: Parameters extracted from fitting a power law to the experimental data. (a) $k$ parameter and (b) $m$ parameter of the power law fitting to the liquid penetration profiles measured by TPI as a function of the nominal porosity, $f_{\text {nominal }}$. The black lines are added to guide the eye. 
the fired alumina samples) is much smaller than the $\mathrm{THz}$ wavelength (around $67 \mu \mathrm{m}$ to $2 \mathrm{~mm}$ ) used in this work, thus very little scattering is expected from such samples.

\subsection{Analysis of the Hydraulic Radius of the Porous Compact}

The hydraulic radius, $R_{\mathrm{h} \text {,eq }}$, of the alumina compact was determined by fitting the Lucas-Washburn equation (Equation 6) to the liquid penetration data measured by TPI. The contact angle between water and the alumina compact with highest compaction force along with the dynamic viscosity and surface tension of water at $20^{\circ} \mathrm{C}\left(1.002 \mathrm{mPas}\right.$ and $72.75 \mathrm{mN} \mathrm{m}^{-1}$, respectively) were used for the calculation of $R_{\mathrm{h} \text {,eq }}$ for each compaction force within each set of firing conditions. Figure 11 shows that the $R_{\mathrm{h}, \text { eq }}$ increases with a decrease in the compaction force, i.e. increase in the porosity, within each set of temperature conditions. Figure 11 also shows that the $R_{\mathrm{h}, \text { eq }}$ is linearly correlated to the median pore radius. The difference in the gradient of this correlation between the $R_{\mathrm{h} \text {,eq }}$ and the median pore radius within each set of temperature conditions can be attributed to differences in the contact angle. An increase in the median pore radius results in an increase in the $R_{\mathrm{h} \text {,eq }}$ which drives the liquid to move faster through the alumina compact.

The TPI method has previously been used by Markl et al. (2018) to investigate the kinetics of water transport into functionalised calcium carbonate (FCC) powder compacts, which are similar to the alumina compacts in the sense that both the FCC and alumina compacts are ceramic materials that do not disintegrate in water due to their rigid, non-dissolving and non-swelling nature in water. The study conducted by Markl et al. (2018) showed that the $k$ parameter obtained from the terahertz liquid transport data of the FCC tablets is linearly correlated to the porosity, and the exponent $m$ shows that mass transport mechanism of such tablets was found to obey Darcy flow. This is consistent with observations in the water transport results of the alumina samples. The $R_{\mathrm{h}, \mathrm{eq}}$ of the FCC tablets was found to have a strong correlation with porosity, which is also the case for the $R_{\mathrm{h} \text {,eq }}$ of the alumina samples within each set of 


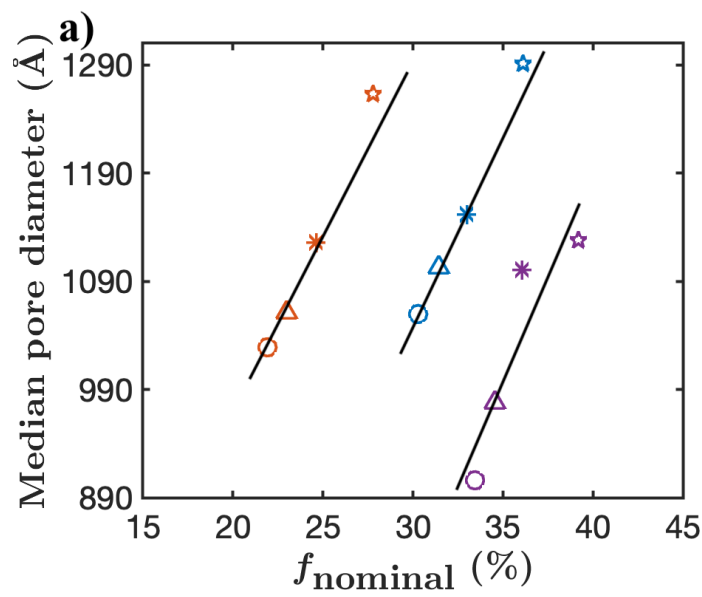

\& Unfired, $07 \mathrm{kN}$

* Unfired, $23 \mathrm{kN}$

$\triangle$ Unfired, $40 \mathrm{kN}$

○ Unfired, $58 \mathrm{kN}$

मे $1200 \mathrm{C}, 07 \mathrm{kN}$

* $1200 \mathrm{C}, 23 \mathrm{kN}$

$\triangle 1200 \mathrm{C}, 40 \mathrm{kN}$

○ $1200 \mathrm{C}, 58 \mathrm{kN}$

น $1300 \mathrm{C}, 07 \mathrm{kN}$

* $1300 \mathrm{C}, 23 \mathrm{kN}$

$\triangle 1300 \mathrm{C}, 40 \mathrm{kN}$

$1300 \mathrm{C}, 58 \mathrm{kN}$

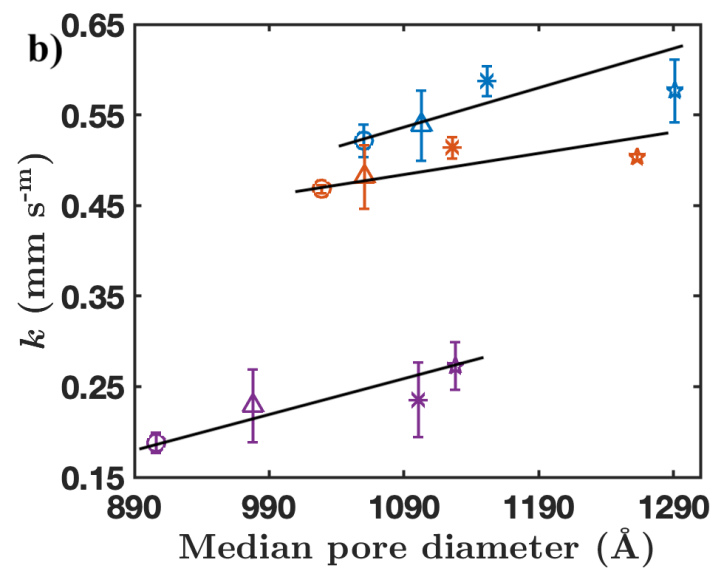

Figure 10: (a) The median pore diameter measured by mercury porosimetry as a function of nominal porosity, $f_{\text {nominal }}$ and (b) the $k$ parameter measured using the TPI method as a function of median pore diameter. The black lines are added to guide the eye. 


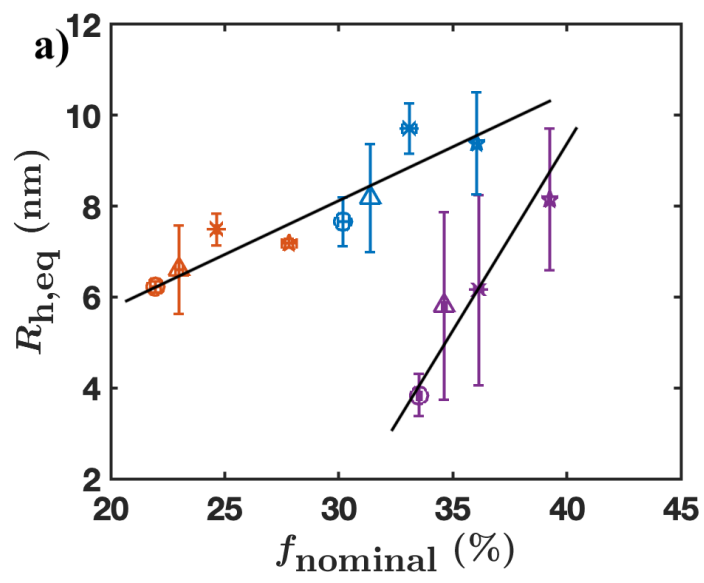

น Unfired, $07 \mathrm{kN}$

* Unfired, $23 \mathrm{kN}$

$\triangle$ Unfired, $40 \mathrm{kN}$

○ Unfired, $58 \mathrm{kN}$

\& $1200 \mathrm{C}, 07 \mathrm{kN}$

* $1200 \mathrm{C}, 23 \mathrm{kN}$

$\triangle 1200 \mathrm{C}, 40 \mathrm{kN}$

○ $1200 \mathrm{C}, 58 \mathrm{kN}$

4 $1300 \mathrm{C}, 07 \mathrm{kN}$

* $1300 \mathrm{C}, 23 \mathrm{kN}$

$\triangle 1300 \mathrm{C}, 40 \mathrm{kN}$

○ $1300 \mathrm{C}, 58 \mathrm{kN}$

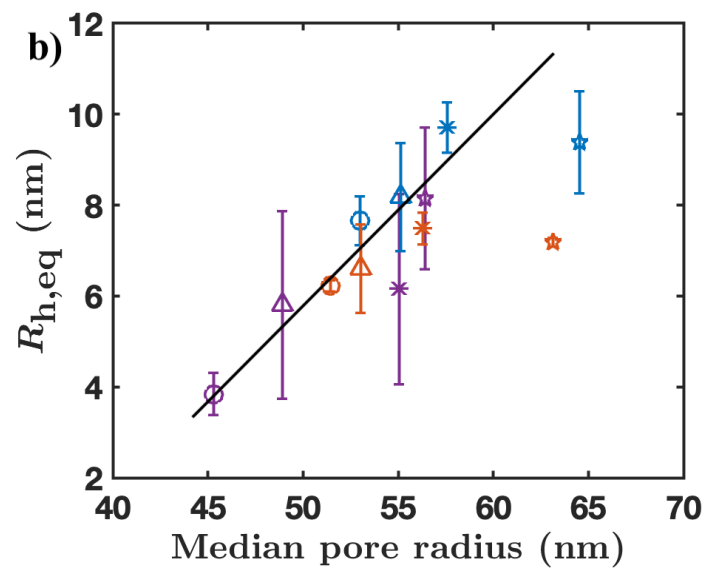

Figure 11: The hydraulic radius, $R_{\mathrm{h}, \text { eq }}$, obtained by fitting the Lucas-Washburn equation to the liquid penetration data acquired by TPI as a function of (a) the nominal porosity, $f_{\text {nominal }}$, and (b) the median pore radius. The black lines are added to guide the eye. 
temperature condition, i.e. unfired and fired. The very small size of the $R_{\mathrm{h}, \text { eq }}$ of the FCC and alumina compacts suggests that the fine structure, i.e. the smallest pores, drive the water penetration at the wetting front, and the imbibition is controlled by the permeability of the compact at long times (Ridgway et al. 2006, Markl et al. 2018).

Sintering is a crucial step in the processing of ceramic powder compacts, such as $\alpha$-alumina, in which the green powder compact is converted into a coherent body with controlled microstructure (Prajzler et al. 2018$)$. The design of strong pellets with sufficient porosity is of great importance to ensure that the pellet does not break upon contact with high pressure liquids during the wetting process. The TPI results (Figure 9) reveal that sintering the green $\alpha$-alumina pellets not only strengthens the pellet and ensures its mechanical stability but also increases its water transport rate when comparing the unfired and fired samples at $1200^{\circ} \mathrm{C}$. Therefore, based on the desired transport kinetics for a specific application, the manufacturing conditions, i.e. compaction force and sintering temperature, can be adjusted carefully so the design of the pellet achieves the target transport kinetics. The results reported in this study show the great potential of using the TPI method for conducting at-line analysis of catalyst manufacturing processes, such as wetting and impregnation, as well as for investigating the impact of the microstructure and surface characteristics on the liquid transport kinetics in a fast manner. The TPI method can further be developed to study the two-dimensional (2D), i.e. axial and radial, liquid transport kinetics of porous ceramic materials as only the 1D liquid transport in the axial direction was investigated. Such results can then be compared to the results of existing methods, such as MRI where the $2 \mathrm{D}$ or $3 \mathrm{D}$ liquid transport into porous ceramics can be investigated. In addition, the current processing of the TPI data that is based on the intensity profile of the time-domain signal for tracking the peak of the liquid front movement can be further extended by reconstructing the primitively profile based on the method by (?). The use of permittivity profile can provide further information on the internal structural changes that occur in the compact during the ingress of the liquid. In summary, 
studying the effect of varying manufacturing conditions on the liquid transport characteristics of catalysts and catalyst supports helps engineers in the catalysis field optimise the design of the catalyst at a reduced cost and controlled quality.

\section{Conclusions}

This work has demonstrated the use of the TPI method in combination with the newly designed flow cell to successfully study the liquid transport kinetics of ceramic catalytic materials. The effect of the microstructure characteristics, such as porosity, as well as the surface properties of the alumina compacts on their liquid transport kinetics has been investigated. It has been shown that the liquid ingress is influenced by both the porosity as well as the surface properties of the alumina pellets. Other microstructure properties of the pellet, such as pore connectivity, can also have an impact on the liquid transport kinetics and therefore it is important to further investigate the impact of such structural properties on the liquid penetration kinetics. The surface properties of the solid material determine its wettability and hence influence its liquid imbibition kinetics. The contact angle results have shown the lower wettability of the unfired samples compared to the fired samples at the same compaction force, which is in agreement with the observations in the terahertz results of the liquid transport kinetics. This shows the potential of using TPI as a new method for conducting at-line analysis of wetting and impregnation of the catalytic materials. The investigation of the effect of both the pore structure characteristics as well as the surface properties of the catalytic materials on their liquid transport kinetics is of great importance for enhancing the design of the catalyst and hence, optimising its performance during heterogenous catalytic reactions where the solid catalyst can come into contact with liquid reactants or products.

Investigating the liquid transport kinetics of catalytic materials is crucial for enhancing the design and hence the performance of such materials. The selection of specific compaction and firing conditions of the catalyst pellet depends on the application. The unfired samples are not strong enough to be used 
in practical applications as such samples might break upon contact with high pressure liquids. Therefore, sintering the pellet is important for ensuring that the pellet is mechanically strong for practical use. Studying the liquid transport kinetics of a combinations of different compaction and firing conditions provides valuable information on the desirable microstructure of the pellet, i.e. a mechanically strong but sufficiently porous pellet, to achieve the desired mass transport kinetics.

\section{Acknowledgements}

We would like to thank Johnson Matthey and the U.K. Engineering and Physical Sciences Research Council (EPSRC) for their funding. We would also like to thank Rob Fletcher from the Johnson Matthey analytical team for conducting the mercury intrusion porosimetry measurements.

\section{References}

\section{References}

Al-Sharabi, M., Markl, D., Mudley, T., Bawuah, P., Karttunen, A.-P., Ridgway, C., Gane, P., Ketolainen, J., Peiponen, K.-E., Rades, T., \& Zeitler, J. A. (2020). Simultaneous investigation of the liquid transport and swelling performance during tablet disintegration. International Journal of Pharmaceutics, 584,119380 .

Anadon, L. D., Lim, M. H. M., Sederman, A. J., \& Gladden, L. F. (2005). Hydrodynamics in two-phase flow within porous media. Magnetic Resonance Imaging, 23, 291-294.

Bej, B., Pradhan, N. C., \& Neogi, S. (2014). Production of hydrogen by steam reforming of ethanol over alumina supported nano-NiO/SiO2 catalyst. Catalysis Today, 237, 80-88. 
Bowker, M., Nuhu, A., \& Soares, J. (2007). High activity supported gold catalysts by incipient wetness impregnation. Catalysis Today, 122, 245-247.

Chen, J., Hu, W., Huang, F., Wu, Y., Yuan, S., Zhong, L., \& Chen, Y. (2018).

$\mathrm{P}$ promotion on the performance of $\mathrm{Pd}$-based catalyst for emission control of natural gas driven vehicles. Journal of the Taiwan Institute of Chemical Engineers, 91, 323-331.

Cummins, B. M., Chinthapatla, R., Ligler, F. S., \& Walker, G. M. (2017). Time-Dependent Model for Fluid Flow in Porous Materials with Multiple Pore Sizes. Analytical Chemistry, 89, 4377-4381.

D’Agostino, C., Kotionova, T., Mitchell, J., Miedziak, P. J., Knight, D. W., Taylor, S. H., Hutchings, G. J., Gladden, L. F., \& Mantle, M. D. (2013). Solvent effect and reactivity trend in the aerobic oxidation of 1,3-propanediols over gold supported on titania: NMR diffusion and relaxation studies. - PubMed - NCBI. Chemistry - A European Journal, 19, 11725-11732.

D’Agostino, C., Mitchell, J., Gladden, L. F., \& Mantle, M. D. (2012). Hydrogen Bonding Network Disruption in Mesoporous Catalyst Supports Probed by PFG-NMR Diffusometry and NMR Relaxometry. The Journal of Physical Chemistry $C, 116,8975-8982$.

Dwivedi, C., Pandey, I., Pandey, H., Ramteke, P. W., Pandey, A. C., Mishra, S. B., \& Patil, S. (2017). Electrospun Nanofibrous Scaffold as a Potential Carrier of Antimicrobial Therapeutics for Diabetic Wound Healing and Tissue Regeneration. In Nano- and Microscale Drug Delivery Systems (pp. 147-164). Elsevier.

Gladden, L. F., Mantle, M. D., \& Sederman, A. J. (2006). Magnetic Resonance Imaging of Catalysts and Catalytic Processes. Advances in Catalysis, 50, $1-75$.

Hornyak, G. L., \& Rao, A. K. (2016). Fundamentals of Nanoscience (and Nanotechnology). In Nanoscience in Dermatology (pp. 15-29). Academic Press. 
Jadhav, S. G., Vaidya, P. D., Bhanage, B. M., \& Joshi, J. B. (2014). Catalytic carbon dioxide hydrogenation to methanol: A review of recent studies. Chemical Engineering Research and Design, 92, 2557-2567.

Jonuscheit, J. (2018). Terahertz Techniques in NDE. In Handbook of Advanced Non-Destructive Evaluation (pp. 1-20). Cham: Springer, Cham.

Jose, A. J., \& Alagar, M. (2015). Preparation and characterization of polysulfone-based nanocomposites. In Manufacturing of Nanocomposites with Engineering Plastics (pp. 31-59). Woodhead Publishing.

Koptyug, I. V., Khitrina, L. Y., Parmon, V. N., \& Sagdeev, R. Z. (2001). NMR imaging of mass transport and related phenomena in porous catalysts and sorbents. Magnetic Resonance Imaging, 19, 531-534.

Kulkarni, V. S., \& Shaw, C. (2016). Surfactants, Lipids, and Surface Chemistry. In Essential Chemistry for Formulators of Semisolid and Liquid Dosages (pp. 5-19). Elsevier.

Leutzsch, M., Sederman, A. J., Gladden, L. F., \& Mantle, M. D. (2019). In situ reaction monitoring in heterogeneous catalysts by a benchtop NMR spectrometer. Magnetic Resonance Imaging, 56, 138-143.

Li, Y., Liu, J., He, J., Wang, L., \& Lei, J. (2020). Silica/titania compositesupported NiCo catalysts with combined catalytic effects for phenol hydrogenation under fast and mild conditions. Applied Catalysis A: General, 591, 117409 .

Louyot, P., Neagoe, C., Galli, F., Pirola, C., Patience, G. S., \& Boffito, D. C. (2018). Ultrasound-assisted impregnation for high temperature FischerTropsch catalysts. Ultrasonics Sonochemistry, 48, 523-531.

Lysova, A. A., Bergwerff, J. A., Espinosa-Alonso, L., Weckhuysen, B. M., \& Koptyug, I. V. (2010). Magnetic resonance imaging as an emerging tool for studying the preparation of supported catalysts. Applied Catalysis A: General, 374, 126-136. 
Lysova, A. A., Koptyug, I. V., Sagdeev, R. Z., Parmon, V. N., Bergwerff, J. A., \& Weckhuysen, B. M. (2005). Noninvasive In Situ Visualization of Supported Catalyst Preparations Using Multinuclear Magnetic Resonance Imaging. Journal of the American Chemical Society, 127, 11916-11917.

Mantle, M. D., Enache, D. I., Nowicka, E., Davies, S. P., Edwards, J. K., D’Agostino, C., Mascarenhas, D. P., Durham, L., Sankar, M., Knight, D. W., Gladden, L. F., Taylor, S. H., \& Hutchings, G. J. (2010). Pulsed-Field Gradient NMR Spectroscopic Studies of Alcohols in Supported Gold Catalysts†. The Journal of Physical Chemistry C, 115, 1073-1079.

Markl, D., Wang, P., Ridgway, C., Karttunen, A.-P., Bawuah, P., Ketolainen, J., Gane, P., Peiponen, K.-E., \& Zeitler, J. A. (2018). Resolving the rapid water absorption of porous functionalised calcium carbonate powder compacts by terahertz pulsed imaging. Chemical Engineering Research and Design, 132, 1082-1090.

Markl, D., Wang, P., Ridgway, C., Karttunen, A.-P., Chakraborty, M., Bawuah, P., Pääkkönen, P., Gane, P., Ketolainen, J., Peiponen, K.-E., \& Zeitler, J. A. (2017a). Characterization of the pore Structure of functionalized calcium carbonate tablets by terahertz time-domain spectroscopy and X-ray computed microtomography. Journal of Pharmaceutical Sciences, 106, 1586-1595.

Markl, D., Yassin, S., Wilson, D. I., Goodwin, D. J., Anderson, A., \& Zeitler, J. A. (2017b). Mathematical modelling of liquid transport in swelling pharmaceutical immediate release tablets. International Journal of Pharmaceutics, $526,1-10$.

Markl, D., \& Zeitler, J. A. (2017). A review of disintegration mechanisms and measurement techniques. Pharmaceutical Research, 34, 890-917.

Nichele, V., Signoretto, M., Menegazzo, F., Gallo, A., Dal Santo, V., Cruciani, G., \& Cerrato, G. (2012). Glycerol steam reforming for hydrogen production: Design of Ni supported catalysts. Applied Catalysis B: Environmental, 111112, 225-232. 
Nijhuis, T. A., Beers, A. E. W., Vergunst, T., Hoek, I., Kapteijn, F., \& Moulijn, J. A. (2001). Preparation of monolithic catalysts. Catalysis Reviews, 43, 345380.

Obradovic, J., Collins, J. H. P., Hirsch, O., Mantle, M. D., Johns, M. L., \& Gladden, L. F. (2007). The use of THz time-domain reflection measurements to investigate solvent diffusion in polymers. Polymer, 48, 3494-3503.

Pickwell, E., \& Wallace, V. P. (2006). Biomedical applications of terahertz technology. Journal of Physics D: Applied Physics, 39, R301-R310.

Prajzler, V., Salamon, D., \& Maca, K. (2018). Pressure-less rapid rate sintering of pre-sintered alumina and zirconia ceramics. Ceramics International, 44, 10840-10846.

Ridgway, C. J., Gane, P. A. C., \& Schoelkopf, J. (2006). Achieving Rapid Absorption and Extensive Liquid Uptake Capacity in Porous Structures by Decoupling Capillarity and Permeability: Nanoporous Modified Calcium Carbonate. Transport in Porous Media, 63, 239-259.

Rytter, E., Borg, Ø., Enger, B. C., \& Holmen, A. (2019). $\alpha$-alumina as catalyst support in Co Fischer-Tropsch synthesis and the effect of added water; encompassing transient effects. Journal of Catalysis, 373, 13-24.

Sankey, M. H., Holland, D. J., Sederman, A. J., \& Gladden, L. F. (2009). Magnetic resonance velocity imaging of liquid and gas two-phase flow in packed beds. Journal of Magnetic Resonance, 196, 142-148.

Schlögl, R. (2015). Heterogeneous catalysis. Angewandte Chemie International Edition, 54, 3465-3520.

Segobia, D. J., Trasarti, A. F., \& Apesteguía, C. R. (2019). Effect of the catalyst preparation method on the performance of Ni-supported catalysts for the synthesis of saturated amines from nitrile hydrogenation. Chinese Journal of Catalysis, 40, 1693-1703. 
Sosna, B., Korup, O., \& Horn, R. (2020). Probing local diffusion and reaction in a porous catalyst pellet. Journal of Catalysis, 381, 285-294.

Stirnimann, T., Di Maiuta, N., Gerard, D. E., Alles, R., Huwyler, J., \& Puchkov, M. (2013). Functionalized calcium carbonate as a novel pharmaceutical excipient for the preparation of orally dispersible tablets. Pharmaceutical Research, 30, 1915-1925.

Washburn, E. W. (1921). The Dynamics of Capillary Flow. Physical Review, $17,273-283$.

Weber, D., Mitchell, J., McGregor, J., \& Gladden, L. F. (2009). Comparing Strengths of Surface Interactions for Reactants and Solvents in Porous Catalysts Using Two-Dimensional NMR Relaxation Correlations. The Journal of Physical Chemistry C, 113, 6610-6615.

Xu, R., Hou, C., Xia, G., Sun, X., Li, M., Nie, H., \& Li, D. (2020). Effects of $\mathrm{Ag}$ promotion for $\mathrm{Co} / \mathrm{Al} 2 \mathrm{O} 3$ catalyst in Fischer-Tropsch synthesis. Catalysis Today, 342, 111-114.

Yassin, S., Goodwin, D. J., Anderson, A., Sibik, J., Wilson, D. I., Gladden, L. F., \& Zeitler, J. A. (2015a). The Disintegration process in microcrystalline cellulose based tablets, part 1: Influence of temperature, porosity and superdisintegrants. Journal of Pharmaceutical Sciences, 104, 3440-3450.

Yassin, S., Su, K., Lin, H., Gladden, L. F., \& Zeitler, J. A. (2015b). Diffusion and swelling measurements in pharmaceutical powder compacts using terahertz pulsed imaging. Journal of Pharmaceutical Sciences, 104, 1658-1667.

Yeboah, Y. D., Ma'adhah, A. G., \& Ali, S. A. (1995). Surface Area and Porosity Measurements of Steam Reforming and Methanation Catalysts. Journal of King Saud University - Engineering Sciences, 7, 257-269.

Zeitler, J. A. (2016). Pharmaceutical Terahertz Spectroscopy and Imaging. In A. Muellertz, Y. Perrie, \& T. Rades (Eds.), Analytical Techniques in the 
Zeitler, J. A., \& Shen, Y.-C. (2012). Industrial Applications of Terahertz Imaging. In K.-E. Peiponen, J. A. Zeitler, \& M. Kuwata-Gonokami (Eds.), Terahertz Spectroscopy and Imaging. Springer Series in Optical Sciences (pp. 451-489). Berlin, Heidelberg: Springer.

Zhokh, A. A., \& Strizhak, P. E. (2017). Effect of zeolite ZSM-5 content on the methanol transport in the ZSM-5/alumina catalysts for methanol-to-olefin reaction. Chemical Engineering Research and Design, 127, 35-44.

Zhou, Y., Lan, G., Zhou, B., Jiang, W., Han, W., Liu, H., \& Li, Y. (2013). Effect of pore structure of mesoporous carbon on its supported Ru catalysts for ammonia synthesis. Chinese Journal of Catalysis, 34, 1395-1401. 


\author{
Appendix A. Supporting Information \\ Appendix A.1. Waterfall Plots of the Fired Alumina Samples at Different Com- \\ paction Forces \\ Appendix A.2. Liquid Penetration Profiles \\ Appendix A.3. Analysis of Liquid Transport Kinetics \\ Appendix A.4. Mercury Porosimetry
}



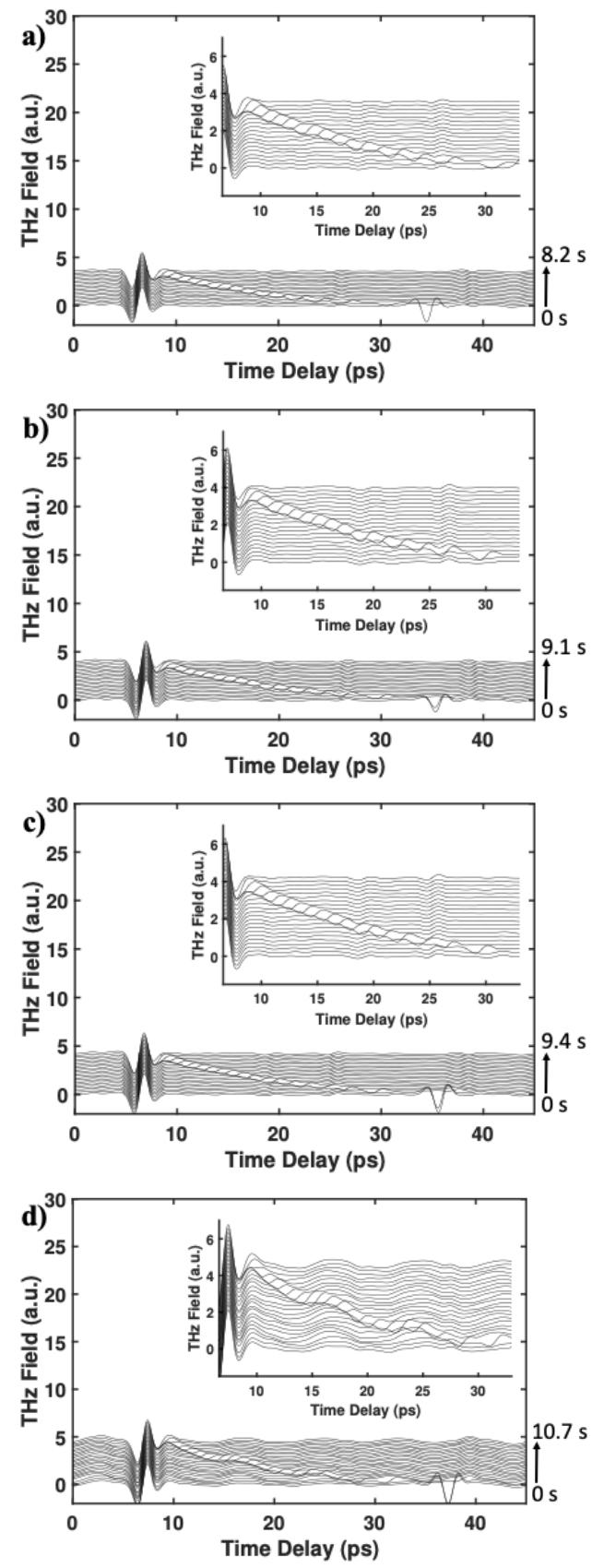

Figure A.12: Waterfall plots visualising the water ingress into the alumina samples fired at $1200{ }^{\circ} \mathrm{C}$ and compacted at different compaction forces of (a) $7 \mathrm{kN}$ (b) $23 \mathrm{kN}$ (c) $40 \mathrm{kN}$ and (d) $58 \mathrm{kN}$. Each seventh deconvolved waveform was plotted with an offset of 0.03 a.u. between each deconvolved waveform. The arrow on the right of each waterfall plot indicates the duration of the full wetting of the sample by water in seconds. 

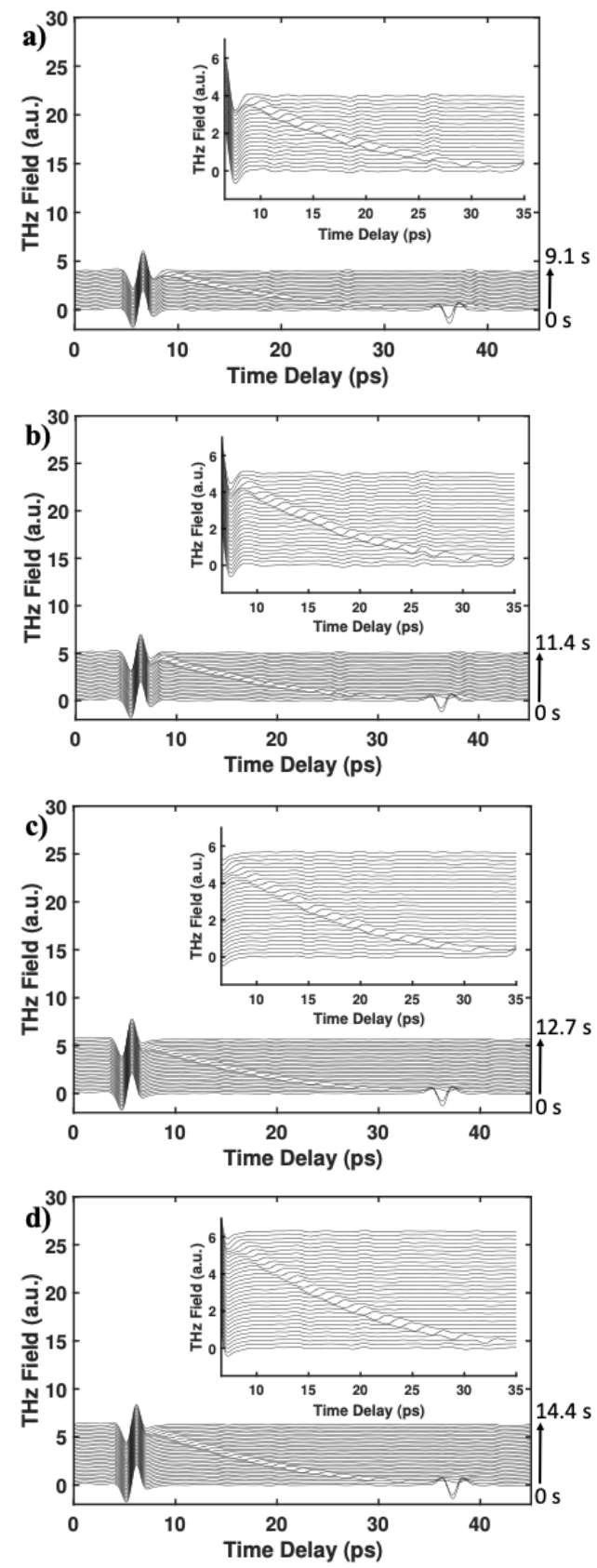

Figure A.13: Waterfall plots visualising the water ingress into the alumina samples fired at $1300{ }^{\circ} \mathrm{C}$ and compacted at different compaction forces of (a) $7 \mathrm{kN}$ (b) $23 \mathrm{kN}$ (c) $40 \mathrm{kN}$ and (d) $58 \mathrm{kN}$. Each seventh deconvolved waveform was plotted with an offset of 0.03 a.u. between each deconvolved waveform. The arrow on the right of each waterfall plot indicates the duration of the full wetting of the sample by water in seconds. 


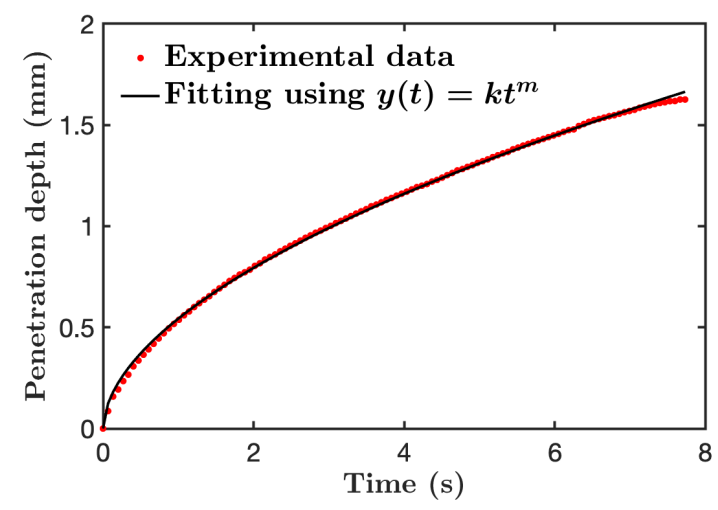

Figure A.15: An illustration of the power law fitting to the experimental liquid penetration data measured by TPI. 


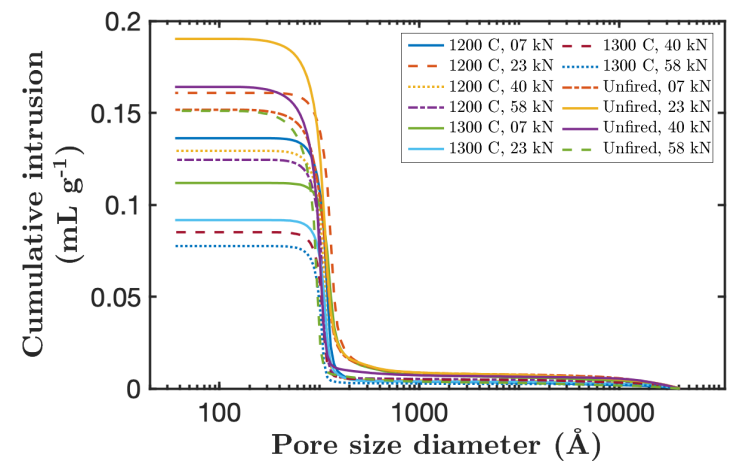

Figure A.16: The cumulative intrusion of the alumina samples as a function of the pore size diameter.

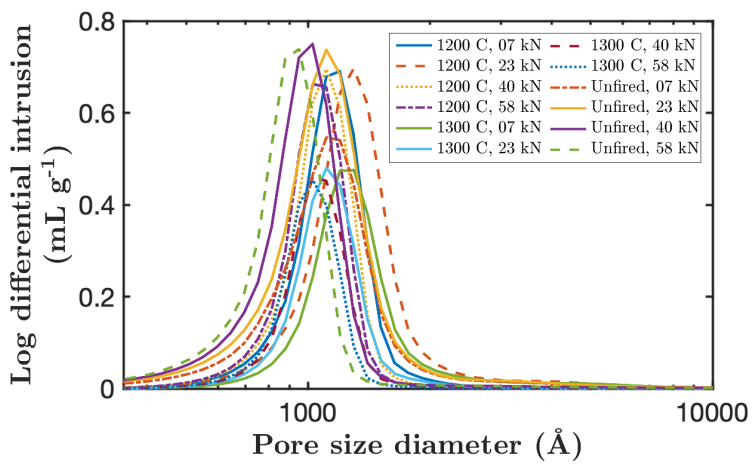

Figure A.17: The log differential intrusion of the alumina samples as a function of the pore size diameter. 